\title{
Taming the Unbounded for Hardware Synthesis
}

\author{
Byron Cook \\ Microsoft Research Cambridge
}

\begin{abstract}
The difficulty with compiling software to hardware is one of finding a priori bounds on the potentially unbounded resources used by programs: memory and time. New approaches now allow us to synthesize bounds on these resources, leading to new high-level hardware synthesis tools.
\end{abstract}

